\title{
Theoretical determination of polarizability and magnetic susceptibility of neon
}

\author{
Michą Lesiuk ** Michą Przybytek, and Bogumį Jeziorski \\ Faculty of Chemistry, University of Warsaw \\ Pasteura 1, 02-093 Warsaw, Poland
}

(Dated: January 27, 2021)

\begin{abstract}
We report theoretical determination of the dipole polarizability of the neon atom, including its frequency dependence. Corrections for the relativistic, quantum electrodynamics, finite nuclear mass, and finite nuclear size effects are taken into account. We obtain the value $\alpha_{0}=2.66080(36)$ for the static polarizability, and $\alpha_{2}=2.850(7)$ and $\alpha_{4}=4.932(14)$ for the first two polarizability dispersion coefficients (Cauchy moments); all values are in atomic units (a.u.). In the case of static polarizability, our result agrees with the best experimental determination [C. Gaiser and B. Fellmuth, Phys. Rev. Lett. 120, 123203 (2018)], but our estimated uncertainty is significantly larger. For the dispersion coefficients, the results obtained in this work appear to be the most accurate to date overall compared to published theoretical and experimental data. We also calculated the static magnetic susceptibility of the neon atom, needed to obtain the refractive index of gaseous neon. Our result, $\chi_{0}=-8.484(19) \cdot 10^{-5}$ a.u., is about $9 \%$ larger in absolute value than the recommended experimental value [CRC Handbook of Chemistry and Physics, CRC Press, 2019, p. 4-145].
\end{abstract}

PACS numbers: 31.15.vn, 03.65.Ge, 02.30.Gp, 02.30.Hq

\section{INTRODUCTION}

Electric dipole polarizability, $\alpha$, is one of the fundamental properties of atomic and molecular systems as it determines response to a perturbation by an external electric field. The importance of polarizability manifests itself also through the Clausius-Mossotti equation

$$
\frac{\epsilon_{r}-1}{\epsilon_{r}+2}=\frac{4 \pi}{3} \alpha \rho
$$

which connects the relative electric permittivity $\epsilon_{r}$ of an atomic gas at low densities $\rho$ with an atomic property. A formula analogous to Eq. (1) holds also for the second important quantity - relative magnetic permeability, $\mu_{r}$. In this case the polarizability in Eq. (1) is replaced by the magnetic susceptibility of the gas, $\chi$. Together, the values of $\epsilon_{r}$ and $\mu_{r}$ determine the refractive index, $n=$ $\sqrt{\epsilon_{r} \mu_{r}}$, which is fundamental in determination of optical properties of materials. The relation (1) is valid only in the low-density limit, but corrections to this equation proportional to higher powers of $\rho$ (expressed through the so-called virial coefficients) are relatively small for noble gases [1].

The connection between the microscopic quantities, $\alpha$ and $\chi$, and the macroscopic ones, $\epsilon_{r}$ and $\mu_{r}$, becomes particularly important if one notices that the latter two are directly accessible by experimental techniques. The relative electric permittivity can be determined from capacitance measurements [2 6] with accuracy reaching one part per million. In fact, such measurements (dielectricconstant gas thermometry) are the source of currently most accurate experimental values of atomic polarizabilities [7]. The refractive index can be measured by several microwave [8] and optical methods [9, 10].

\footnotetext{
* e-mail: lesiuk@tiger.chem.uw.edu.pl
}

Development of new experimental techniques [2 10 has been critical for the progress in modern thermal metrology. For example, it has been proposed to establish a new pressure standard based on the measurements of $\epsilon_{r}$, or on laser refractometry measurements of the refractive index [10]. The link between pressure $p$ of the gas and the refractive index $n$ is provided by the following equation

$$
p=\frac{k T}{2 \pi(\alpha+\chi)}(n-1),
$$

valid in the low-pressure limit. Since the value of the Boltzmann constant $k$ is now fixed according to the new SI definition of the unit of temperature $T$ [11, 12], the knowledge of $\alpha$ and $\chi$ is required to obtain $p$ directly from $n$, via Eq. (2).

A significant portion of the experimental effort in this field targets noble gases due to numerous favorable properties such as being chemically inert and stable, etc. The simplest member of this family, i.e., helium, has been intensively studied in recent years also by theoretical methods 13 20]. The static dipole polarizability of the helium atom is now known from theory with relative accuracy of $10^{-7}$ [21]. This is more than sufficient for the purposes of metrology. However, the same cannot be said about the heavier noble gas atoms. For example, most systematic theoretical studies of the polarizability of the neon atom were undertaken more than a decade ago [22 29]. Additionally, if one aims at the accuracy better than one part per thousand it is necessary to include various corrections accounting for effects beyond the clamped-nuclei nonrelativistic Schrödinger equation which has not been systematically done thus far.

The main purpose of the present work is state-of-theart theoretical determination of the static and dynamic polarizability of the neon atom at experimentally relevant frequencies. To this end, we employ high-level $a b i n i$ tio electronic structure methods and basis sets designed 
specifically for the task. Our study is not limited to the nonrelativistic picture and we consider corrections that account for other relevant physical effects. This includes relativistic, quantum electrodynamics (QED), finite nuclear mass, and finite nuclear size effects.

We also compute the magnetic susceptibility $\chi$ relevant for the measurements of the refractive index. Since $\chi$ is about five orders of magnitude smaller than $\alpha$, it does not have to be known with very high accuracy when used in Eq. (2). Therefore, we determine $\chi$ using the nonrelativistic theory. An estimate of the neglected relativistic and QED effects is included in the final uncertainty estimate.

Atomic units (a.u.) are used throughout the present work unless explicitly stated otherwise. The following values of physical constants are assumed: speed of light in vacuum, $c=137.035999084$, proton-toelectron mass ratio, $m_{\mathrm{p}} / m_{\mathrm{e}}=1836.15267343$, Bohr radius, $a_{0}=0.529177210903 \AA$, Avogadro number, $N_{A}=$ $6.02214076 \cdot 10^{23}$, according to the most recent CODATA database [30]. All results given in this paper refer to the most abundant ${ }^{20} \mathrm{Ne}$ isotope of neon with the nuclear mass equal to $36434.0 m_{\mathrm{e}}$ [31].

\section{THEORETICAL CALCULATIONS}

\section{A. Clamped-nucleus nonrelativistic polarizability}

Polarizability is a quantity that depends on the frequency of the external electric field $\omega$ that perturbs the system. This frequency dependence is non-negligible in accurate treatments and must be taken into account in calculation of quantities appearing in Eq. (11). Assuming that the necessary range of frequencies corresponds to energies well below the first atomic resonant frequency [32] ( $\omega \approx 0.6107 \mathrm{a} . \mathrm{u}$ ), we can use the following expansion

$$
\alpha(\omega)=\alpha_{0}+\alpha_{2} \omega^{2}+\alpha_{4} \omega^{4}+\ldots
$$

where $\alpha_{2}, \alpha_{4}, \ldots$ are the so-called dispersion coefficients (Cauchy coefficients) and $\alpha_{0}:=\alpha(0)$ is a shorthand notation for the static polarizability.

For frequencies that are of particular experimental interest, e.g., helium-neon laser operating at wavelengths near $633 \mathrm{~nm}$ (which corresponds to $\omega \approx 0.072$ a.u.), the expansion (31) is rapidly convergent. The inclusion of terms only up to $\omega^{4}$ is sufficient to reach relative accuracy in $\alpha(\omega)$ better than $10^{-6}$ for the neon atom at this frequency. By retaining only the quadratic term in Eq. (3), the $10^{-4}$ accuracy level is attainable. Moreover, assuming that $\alpha_{0}$ was calculated with relative accuracy of about $10^{-5}$ and the same accuracy level is to be retained in $\alpha(\omega)$, the coefficient $\alpha_{2}$ must be accurate to a few parts per thousand, and the coefficient $\alpha_{4}$ to only about $10 \%$. This greatly simplifies the incorporation of frequency dependence into the theoretical results.

The first step in the calculations is an accurate determination of the nonrelativistic polarizability of the neon atom with inclusion of the frequency-dependence, i.e. $\alpha_{0}$, $\alpha_{2}$, and $\alpha_{4}$. These calculations were performed using the basis sets of Slater-type orbitals (STO) [33, 34] optimized specifically for the purposes of the present work. The basis sets are designated wtcc $X$ (well-tempered correlationconsistent) where $X=2, \ldots, 7$, referred usually to as the cardinal number, stands for the highest angular momentum $l$ included in the basis. They are designed according to the correlation-consistency principle [35] which allows for a reliable extrapolation towards the complete basis set limit (the basis wtcc2 has the composition $6 s 4 p 1 d$ and each consecutive basis has one more function for each angular momentum $l \leq X$ ). To properly allow for the polarization by the electric field, these bases are augmented by adding diffuse functions (with small exponents). The notation "no-aug" means no augmentation, while "s-aug", "d-aug", "t-aug", and "q-aug" stand for the inclusion of one, two, three, and four diffuse functions for each angular momentum. These bases, optimized using the methodology established in Refs. [36, 37], are available from authors upon request. Computer codes for the STO integrals, described in Refs. [38 [40], were used in all relevant calculations. Most calculations reported in this paper were accomplished using variants of the coupled cluster (CC) theory; for a detailed review of this family of methods see Ref. [41].

To test the quality of the basis sets developed in this work, the static polarizability of the helium atom was calculated. The optimization and composition of the helium basis sets is fully analogous to the ones described above the only difference is that the number of the $s$ functions is smaller by one at each augmentation and polarization level. The helium atom constitutes an excellent benchmark for the purposes of this work since a practically exact value of the non-relativistic clamped-nuclei static polarizability is known, $\alpha_{0}(\mathrm{He})=1.383192174455(1)$, as reported by Pachucki and Sapirstein [15]. Various corrections beyond this level of theory were also reported in the literature [15, 17, 20, 21, 42, 43].

In Table 【 the static polarizability of helium atom calculated using the coupled cluster theory with single and double substitutions [44, 45] (CCSD) is presented. Since for two-electron systems the CCSD method is equivalent to the full configuration interaction (FCI), the only source of error in these results (compared with the reference value cited above) is the incompleteness of the basis set. While the results are well-saturated with respect to the augmentation level, the same is not true when considering the maximum angular momentum $l=X$ included in the basis set. To circumvent this problem and to improve the convergence with increasing cardinal number $X$, the results were extrapolated by using the conventional $X^{-3}$ formula [6 48]. The value extrapolated from two consecutive basis sets $(X$ and $X+1)$ shall be denoted $\operatorname{CBS}(X, X+1)$ where the abbreviation CBS stands for the complete basis set limit. For simplicity, extrapolation with respect to the augmentation level was not attempted and q-aug basis sets were used throughout. 
TABLE I. Linear-response CCSD static polarizability $\alpha_{0}$ calculated for the helium atom.

\begin{tabular}{ccccccc}
\hline \hline & wtcc2 & wtcc3 & wtcc4 & wtcc5 & wtcc6 & wtcc7 \\
\hline no-aug & 1.37181 & 1.37576 & 1.37886 & 1.38118 & 1.38198 & 1.38253 \\
s-aug & 1.38402 & 1.38345 & 1.38335 & 1.38355 & 1.38344 & 1.38339 \\
d-aug & 1.38414 & 1.38384 & 1.38354 & 1.38365 & 1.38350 & 1.38341 \\
t-aug & 1.38401 & 1.38388 & 1.38358 & 1.38366 & 1.38350 & 1.38340 \\
q-aug & 1.38392 & 1.38390 & 1.38359 & 1.38367 & 1.38350 & 1.38340 \\
\hline \hline
\end{tabular}

TABLE II. Linear-response CC3 static polarizability $\alpha_{0}$ calculated for the neon atom with all electrons correlated.

\begin{tabular}{ccccccc}
\hline \hline & wtcc2 & wtcc3 & wtcc4 & wtcc5 & wtcc6 & wtcc7 \\
\hline no-aug & 0.81910 & 1.49892 & 1.60219 & 1.99140 & 2.13575 & 2.26198 \\
s-aug & 2.43166 & 2.33238 & 2.49043 & 2.55603 & 2.58556 & 2.60764 \\
d-aug & 2.65436 & 2.65557 & 2.67004 & 2.66564 & 2.66406 & 2.66294 \\
t-aug & 2.66676 & 2.68160 & 2.67124 & 2.66674 & 2.66474 & 2.66362 \\
q-aug & 2.66926 & 2.68142 & 2.67115 & 2.66678 & 2.66473 & 2.66361 \\
\hline \hline
\end{tabular}

The results of the performed extrapolations are

$$
\begin{aligned}
& \operatorname{CBS}(3,4)=1.38337, \\
& \operatorname{CBS}(4,5)=1.38374, \\
& \operatorname{CBS}(5,6)=1.38326, \\
& \operatorname{CBS}(6,7)=1.38322 .
\end{aligned}
$$

It is reasonable to assume that the error of the last result is no larger than the difference between the $\operatorname{CBS}(5,6)$ and CBS $(6,7)$ extrapolations. This gives our estimation for the non-relativistic clamped-nuclei static polarizability of the helium atom, $\alpha_{0}(\mathrm{He})=1.38322(4)$. Compared with the reference value given above, the true error of this result is about 2 parts per $10^{5}$. Moreover, the reference value lies within the error bars estimated by us.

Our workhorse method for determination of the nonrelativistic clamped-nuclei polarizability is the orbitalunrelaxed linear-response CC3 method as implemented in the Dalton program package [25, 26, 49, 50]. For the purposes of this work, the STO integral code was interfaced with the DALTON program. The CC3 method is an advanced approximate variant of the coupled-cluster method designed to include the major part of the contribution of three-electron excitations in a computationally efficient way. The CC3 polarizability is calculated with help of the linear response function obtained from time-dependent quasienergy Lagrangian. The resulting equations for the triply excited component of the linear response function are then truncated at the second order in the fluctuation potential enabling an efficient evaluation, see Ref. 25] for a complete presentation.

In Table II , we present CC3 results of the static polarizability of the neon atom. The values converge rather quickly with the augmentation level, practically as fast as in the case of the helium atom. For example, in all basis sets other than the smallest ones, the transition from t-aug to q-aug basis sets changes the results only at the sixth significant digit. Therefore, the larger triplyaugmented basis sets can be viewed as saturated with respect to the augmentation level. Unfortunately, the convergence is not as rapid with respect to the increasing angular momentum in the basis set. To remedy this problem we extrapolated the results to the complete basis set limit by using the same strategy as for the helium atom. Extrapolations of the results from the q-aug basis sets give

$$
\begin{aligned}
& \operatorname{CBS}(3,4)=2.66365, \\
& \operatorname{CBS}(4,5)=2.66220, \\
& \operatorname{CBS}(5,6)=2.66190, \\
& \operatorname{CBS}(6,7)=2.66172 .
\end{aligned}
$$

One can see that the extrapolated results are very stable and that the convergence towards the limit is markedly improved. While the estimation of errors shall be a subject of further discussion, observing the convergence pattern of the extrapolated results allows us to suggest that the value $\alpha_{0}=2.66172(18)$ is a reasonable estimate for the basis set limit of the CC3 static polarizability of the neon atom. The estimated error of this result is thus about 7 parts per $10^{5}$, about 3.5 times larger than in the case of the helium atom.

Although all electrons were correlated to obtain the results presented in Table 【, it is of interest to see what is the contribution to $\alpha_{0}$ coming from the core $\left(1 s^{2}\right)$ and core-valence correlation. In Table【I, we show the difference between CC3 polarizabilities calculated with frozen core and with all electrons correlated. It turns out that the effect of the core and core-valence correlation is relatively small and stabilizes more rapidly with the size of the basis set than the all-electron results reported in Table II. By extrapolating the contributions from Table III obtained with the q-aug basis sets, we find

$$
\begin{aligned}
& \operatorname{CBS}(4,5)=0.00644, \\
& \operatorname{CBS}(5,6)=0.00637 .
\end{aligned}
$$

so that the final estimate for the core contribution to the static polarizability of the neon atom is $0.00637(7)$. This constitutes only about $2 \%$ of the total CC3 correlation contribution to the static polarizability. We assume it is

TABLE III. Core contribution to the static polarizability $\alpha_{0}$ of the neon atom calculated at the CC3 level of theory.

\begin{tabular}{cccccc}
\hline \hline & wtcc2 & wtcc3 & wtcc4 & wtcc5 & wtcc6 \\
\hline no-aug & 0.00016 & 0.00104 & 0.00165 & 0.00322 & 0.00389 \\
s-aug & 0.00210 & 0.00325 & 0.00434 & 0.00522 & 0.00560 \\
d-aug & 0.00295 & 0.00457 & 0.00527 & 0.00584 & 0.00607 \\
t-aug & 0.00300 & 0.00492 & 0.00534 & 0.00587 & 0.00608 \\
q-aug & 0.00302 & 0.00503 & 0.00537 & 0.00589 & 0.00609 \\
\hline \hline
\end{tabular}


TABLE IV. Post-CC3 triples and quadruples corrections to the static polarizability, $\alpha_{0}$, of the neon atom (frozen $1 s$ core orbital).

\begin{tabular}{|c|c|c|c|c|}
\hline & cc-pVDZ & cc-pVTZ & cc-pVQZ & cc-pV5Z \\
\hline & \multicolumn{4}{|c|}{ CCSDT - CC3 correction } \\
\hline s-aug & 0.00137 & 0.00212 & 0.00142 & 0.00079 \\
\hline d-aug & 0.00119 & 0.00119 & 0.00065 & 0.00050 \\
\hline \multirow[t]{2}{*}{ t-aug } & 0.00115 & 0.00109 & 0.00063 & 0.00049 \\
\hline & \multicolumn{4}{|c|}{ full Q correction } \\
\hline s-aug & -0.00222 & -0.00422 & -0.00489 & - \\
\hline d-aug & -0.00542 & -0.00597 & - & - \\
\hline t-aug & -0.00555 & -0.00597 & - & — \\
\hline
\end{tabular}

unlikely that this ratio increases substantially in calculation of higher-order correlation effects and use the results of Table III to justify the neglect of core contribution to some higher-order correlation effects discussed further in the text.

The CC3 method is an approximate model which misses some of the effects of the triple excitations and neglects higher excitations entirely. Therefore, it is necessary to account for these effects using a higher-level theory. The complete post-CC3 contribution to the polarizability is split into three components

- post-CC3 triples contribution, i.e., the difference between the coupled cluster method with single, double and triple excitations [51, 52] (CCSDT) and the CC3 results;

- full quadruples contribution, i.e., the difference between the coupled cluster method with single, double, triple and quadruple excitations [53, 54] (CCSDTQ) and the CCSDT results;

- post-CCSDTQ contributions, i.e., the difference between the FCI and CCSDTQ results.

We also explored the possibility of using methods that account for the quadruples perturbatively, such as CCSDT[Q] [55] and $\operatorname{CCSDT}(\mathrm{Q})$ [56]. However, we found them to be very unreliable in (finite-field) calculation of properties. For this reason the use of perturbative quadruple models was abandoned.

In CCSDT and CCSDTQ calculations, we employed the aug-cc-pVXZ Gaussian basis set family, $X=2-5$ [35, 57]. To saturate the results with respect to the augmentation level, we additionally created the doublyaugmented (d-aug-cc-pVXZ) and triply-augmented (taug-cc-pVXZ) variants. They were obtained by scaling the smallest exponent of the Gaussian function in a given shell by the ratio of the smallest two. The CCSDT and CCSDTQ polarizabilities were calculated analytically (without orbital relaxation) with help of the MRCC program package 58. To reduce the computational burden, we froze the $1 s^{2}$ core of the neon atom; as discussed above this approximation is accurate to $1-2 \%$ and thus entirely sufficient for the present purposes.

The full T and Q corrections to the static polarizability of the neon atom are given in Table IV Extrapolation of the full triples correction using the results from the t-aug basis sets gives

$$
\begin{aligned}
& \operatorname{CBS}(3,4)=0.00029, \\
& \operatorname{CBS}(4,5)=0.00034,
\end{aligned}
$$

and an extrapolation with respect to the augmentation level does not seem to be necessary. This allows us to estimate that the full triples correction amounts to $0.00034(5)$, where the uncertainty is the difference between the last two extrapolated values. Moving to the quadruples correction, the same procedure gives only one value

$$
\operatorname{CBS}(2,3)=-0.00614
$$

and our final estimation of this correction is $-0.00614(17)$, where the error is the difference between the extrapolated value and the result obtained with the t-aug-cc-pVTZ basis. The results shown in Table IV show that the $\mathrm{T}$ and $\mathrm{Q}$ corrections to the static polarizability of the neon atom are of the order of a few parts per thousand, and thus are non-negligible from the present point of view. To account for even higher-order excitations we employ the FCI method using dedicated codes written by one of us [59]. This is very computationally expensive and we managed to calculate the FCI correction only in smaller basis sets. Since no extrapolation can be performed we simply add the value calculated with the d-aug-cc-pVDZ (the largest basis set for which the FCI result could be obtained) and assign $50 \%$ uncertainty to it, getting $-0.00047(24)$. It is worth pointing out that even within this relatively small basis, the number of configurations that were included in the FCI calculations reached about $2 \cdot 10^{9}$.

\begin{tabular}{|c|c|c|c|c|c|c|}
\hline & wtcc 2 & wtcc3 & wtcc 4 & wtcc5 & wtcc6 & wtcc7 \\
\hline \multicolumn{7}{|c|}{$\alpha_{2}$} \\
\hline no-aug & 0.20491 & 0.90963 & 0.66040 & 1.52304 & 1.82238 & 1.94098 \\
\hline s-aug & 1.92647 & 2.00067 & 2.14567 & 2.40571 & 2.49805 & 2.57002 \\
\hline d-aug & 2.93317 & 2.72236 & 314 & 2.84109 & 2.84239 & 2.83986 \\
\hline t-aug & 2.94922 & 2.90711 & 2.87749 & 2.86330 & 2.85852 & 2.85389 \\
\hline q-aug & 2.95149 & 2.91005 & 2.87799 & 2.86392 & 2.85861 & 2.85398 \\
\hline \multicolumn{7}{|c|}{$\alpha_{4}$} \\
\hline no-aug & 0.08712 & 1.07861 & 0.48510 & 2.36422 & 3.45964 & 3.52939 \\
\hline s-aug & 2.66424 & 3.42031 & 3.11409 & 4.02005 & 4.13486 & 4.24444 \\
\hline d-aug & 5.39007 & 4.57093 & 4.85203 & 4.85022 & 4.85212 & 4.85396 \\
\hline t-aug & 5.34708 & 5.08456 & 5.00535 & 4.96669 & 4.94596 & 4.93349 \\
\hline q-aug & 5.33855 & 5.11252 & 5.00519 & 4.96531 & 4.94504 & 4.93266 \\
\hline
\end{tabular}

TABLE V . Linear-response CC3 dispersion coefficients $\alpha_{2}$ and $\alpha_{4}$ calculated for the neon atom with all electrons correlated. 
Results of the calculations of the dispersion coefficients $\alpha_{2}$ and $\alpha_{4}$, performed with the all-electron CC3 method are given in Table V] Extrapolation of these data by using the same scheme as for the static polarizability gives

$$
\alpha_{2}=2.846(5), \quad \alpha_{4}=4.912(6) .
$$

Computation of the post-CC3 and Q corrections to the dispersion coefficients is very complex and is not implemented in quantum chemistry software available to us. Therefore, we directly correct for all effects beyond the CC3 model by using the FCI calculations in the d-augcc-pVDZ basis set using a program written specifically for this purpose 59]. Similarly as before, a very conservative accuracy estimate of $50 \%$ is used for this quantity, giving $-0.0089(44)$ and $-0.026(13)$ contributions to $\alpha_{2}$ and $\alpha_{4}$, respectively.

\section{B. Relativistic and QED corrections to the polarizability}

In order to calculate the static polarizability of the neon atom with the accuracy better than a few parts per thousand, one has to consider a number of corrections accounting for various effects beyond the non-relativistic clamped-nuclei Schrödinger equation. The list consists of

1. relativistic correction of the order $1 / c^{2}$,

2. leading-order $\left(1 / c^{3}\right)$ quantum electrodynamics (QED) correction,

3. finite nuclear mass (FNM) correction,

4. finite nuclear size (FNS) correction,

5. higher-order $\left(1 / c^{4}\right.$ and higher $)$ relativistic and QED effects,

where $c$ denotes the speed of light in vacuum (employed instead of the fine-structure constant to avoid a notational collision).

Calculation of the relativistic corrections is based on the Breit-Pauli Hamiltonian 60]

$$
\hat{H}_{\mathrm{BP}}=\hat{P}_{4}+\hat{D}_{1}+\hat{D}_{2}+\hat{B}
$$

where the individual operators are defined as

$$
\begin{gathered}
\hat{P}_{4}=-\frac{1}{8 c^{2}} \sum_{i} \nabla_{i}^{4}, \\
\hat{D}_{1}=\frac{\pi}{2 c^{2}} Z \sum_{i} \delta\left(\mathbf{r}_{i a}\right), \\
\hat{D}_{2}=\frac{\pi}{c^{2}} \sum_{i>j} \delta\left(\mathbf{r}_{i j}\right),
\end{gathered}
$$

$$
\hat{B}=\frac{1}{2 c^{2}} \sum_{i>j}\left[\frac{\nabla_{i} \cdot \nabla_{j}}{r_{i j}}+\frac{\mathbf{r}_{i j} \cdot\left(\mathbf{r}_{i j} \cdot \nabla_{j}\right) \nabla_{i}}{r_{i j}^{3}}\right],
$$

where $Z$ is the nuclear charge. Relativistic corrections to $\alpha_{n}, n=0,2,4$, due to the operators $X$ of Eqs. (5) (8), will be denoted by $\alpha_{n}^{(2)}(X)$. The explicit expression for $\alpha_{n}^{(2)}(X)$ can be obtained by adding $\lambda X$ to the nonrelativistic Hamiltonian, where $\lambda$ is a formal parameter, evaluating the polarizability $\alpha_{n}(X)$, and extracting terms linear in $\lambda$.

The explicit formulas for $\alpha_{n}^{(2)}(X)$ are known [19], but are difficult to implement in practice for many-electron systems. Therefore, we adopted a finite-field [for $\alpha_{0}^{(2)}(X)$ ] or finite-difference approach [for $\left.\alpha_{n}^{(2)}(X), n \geq 2\right]$. In the case of static polarizability, the expectation values of the operators from Eqs. (5)-(8) were evaluated analytically by the linear response approach 61] and then numerically differentiated twice with respect to the strength of the applied external electric field. The electric-field strengths in the interval $0.0-0.005$ were tested and in all cases at least two significant digits were stable in the final results. These calculations were performed with the help of the DALTON program package using the orbital-relaxed CCSD theory with perturbative treatment of triple excitations $[\mathrm{CCSD}(\mathrm{T})]$ as described in Ref. 61]. This method of evaluating CC properties is based on the so-called CC Lagrangian which, in contrast to the standard CC energy expression, is variational with respect to all wavefunction parameters [62 65]. This enables to obtain firstorder properties using a generalization of the HellmannFeynman theorem.

For the dispersion coefficients $\alpha_{2}$ and $\alpha_{4}$, we neglected the two-electron relativistic corrections, i.e., $\hat{D}_{2}$ and $\hat{B}$, since the accuracy requirements are less stringent in this case. Since differentiation with respect to the external field is not applicable for the dispersion coefficients, a different approach was adopted. The operators $\hat{P}_{4}$ and $\hat{D}_{1}$ multiplied by a formal parameter $\lambda$ were added to the Hamiltonian and then the dispersion coefficients were calculated analytically using the CC3 level of theory. The values of the relativistic corrections $\alpha_{0}^{(2)}\left(\hat{P}_{4}\right)$ and $\alpha_{0}^{(2)}\left(\hat{D}_{1}\right)$ were extracted by computing the first derivative with respect to $\lambda$ employing two-point finite-difference formula. The results were sufficiently stable for $\lambda$ within the range $5 \cdot 10^{-5}-1 \cdot 10^{-3}$.

The one-electron relativistic corrections to the polarizabilities and dispersion coefficients were obtained by using a modified Slater-type basis sets - a common set of $20 s 15 p$ functions (obtained by minimization of the atomic Hartree-Fock energy) was used in all basis sets instead of the original sets used in the nonrelativistic calculations. The remaining polarization functions were unchanged.

The computed one-electron relativistic corrections to the static polarizability are shown in Table VI It is seen that the convergence to the basis set limit is rather slow but small basis sets already give accurate results due to 
TABLE VI. One-electron relativistic corrections to the static polarizability of the neon atom calculated at the CCSD(T) level of theory with the finite-field approach.

\begin{tabular}{|c|c|c|c|c|c|c|c|c|c|}
\hline \multirow[t]{2}{*}{ basis set } & \multicolumn{3}{|c|}{ s-aug } & \multicolumn{3}{|c|}{ d-aug } & \multicolumn{3}{|c|}{ t-aug } \\
\hline & $\alpha_{0}^{(2)}\left(P_{4}\right)$ & $\alpha_{0}^{(2)}\left(D_{1}\right)$ & sum & $\alpha_{0}^{(2)}\left(P_{4}\right)$ & $\alpha_{0}^{(2)}\left(D_{1}\right)$ & sum & $\alpha_{0}^{(2)}\left(P_{4}\right)$ & $\alpha_{0}^{(2)}\left(D_{1}\right)$ & sum \\
\hline wtcc $2 /$ sp & 0.01826 & -0.01465 & 0.00360 & 0.02183 & -0.01749 & 0.00434 & 0.02195 & -0.01758 & 0.00436 \\
\hline wtcc3/sp & 0.01748 & -0.01395 & 0.00353 & 0.02114 & -0.01701 & 0.00413 & 0.02181 & -0.01752 & 0.00429 \\
\hline wtcc $4 / \mathrm{sp}$ & 0.01893 & -0.01521 & 0.00372 & 0.02153 & -0.01732 & 0.00422 & 0.02160 & -0.01736 & 0.00423 \\
\hline wtcc5/sp & 0.01965 & -0.01580 & 0.00385 & 0.02147 & -0.01728 & 0.00420 & 0.02152 & -0.01731 & 0.00421 \\
\hline wtcc6/sp & 0.02004 & -0.01613 & 0.00391 & 0.02145 & -0.01726 & 0.00419 & 0.02149 & -0.01729 & 0.00420 \\
\hline
\end{tabular}

TABLE VII. One-electron relativistic corrections to the dispersion coefficients $\alpha_{2}$ and $\alpha_{4}$ calculated at the CC3 level of theory with the finite-difference approach.

\begin{tabular}{|c|c|c|c|c|c|c|c|c|c|}
\hline \multirow[t]{2}{*}{ basis set } & \multicolumn{3}{|c|}{ s-aug } & \multicolumn{3}{|c|}{ d-aug } & \multicolumn{3}{|c|}{ t-aug } \\
\hline & $\alpha_{n}^{(2)}\left(P_{4}\right)$ & $\alpha_{n}^{(2)}\left(D_{1}\right)$ & sum & $\alpha_{n}^{(2)}\left(P_{4}\right)$ & $\alpha_{n}^{(2)}\left(D_{1}\right)$ & sum & $\alpha_{n}^{(2)}\left(P_{4}\right)$ & $\alpha_{n}^{(2)}\left(D_{1}\right)$ & sum \\
\hline \multicolumn{10}{|c|}{$\alpha_{2}$} \\
\hline wtcc $2 /$ sp & 0.04789 & -0.03461 & 0.01328 & 0.06868 & -0.05059 & 0.01809 & 0.06875 & -0.05068 & 0.01807 \\
\hline wtcc3/sp & 0.03971 & -0.03623 & 0.00348 & 0.06219 & -0.04596 & 0.01623 & 0.06699 & -0.04942 & 0.01757 \\
\hline $\mathrm{wtcc} 4 / \mathrm{sp}$ & 0.05169 & -0.03789 & 0.01380 & 0.06275 & -0.04810 & 0.01466 & 0.06508 & -0.04906 & 0.01602 \\
\hline wtcc5/sp & 0.05453 & -0.04147 & 0.01306 & 0.06268 & -0.04803 & 0.01466 & 0.06354 & -0.04849 & 0.01506 \\
\hline \multicolumn{10}{|c|}{$\alpha_{4}$} \\
\hline wtcc $2 /$ sp & 0.11947 & -0.08409 & 0.03538 & 0.19957 & -0.14602 & 0.05355 & 0.19769 & -0.14520 & 0.05249 \\
\hline wtcc3/sp & 0.13860 & -0.10181 & 0.03680 & 0.17670 & -0.12892 & 0.04778 & 0.19043 & -0.13924 & 0.05119 \\
\hline wtcc $4 /$ sp & 0.13331 & -0.09515 & 0.03816 & 0.17784 & -0.13225 & 0.04559 & 0.18435 & -0.13729 & 0.04706 \\
\hline wtcc5/sp & 0.15610 & -0.11765 & 0.03844 & 0.17568 & -0.13248 & 0.04321 & 0.18216 & -0.13528 & 0.04688 \\
\hline
\end{tabular}

the presence of the common $20 s 15 p$ functions. Based on the values provided in Table VI, it can be assumed that

$$
\alpha_{0}^{(2)}\left(P_{4}\right)=0.02149(10), \quad \alpha_{0}^{(2)}\left(D_{1}\right)=-0.01729(10),
$$

where the uncertainties account for the basis set incompleteness error and for the error due to the finite-field approach. Note that there is a significant cancellation between the contributions from the $\hat{P}_{4}$ and $\hat{D}_{1}$ operators. Because of that the error in the sum of P4 and D1 corrections is much smaller than in the individual components. The final one-electron relativistic correction to the atomic polarizability of the neon atom is thus equal to $0.00420(5)$, where error reduction by a factor of two with respect to the $\mathrm{P} 4$ and $\mathrm{D} 1$ corrections was obtained. This value compares quite well with the values 0.00432 , 0.00428 , and 0.00443 (depending on the basis set) obtained by Klopper et al. [22] employing the so called direct perturbation theory (DPT) 66, 67] at the $\operatorname{CCSD}(\mathrm{T})$ level.

The corresponding results for the dispersion coefficients are given in Table VII Note that the convergence of the results is somewhat erratic in basis sets with low augmentation levels. Fortunately, for triply-augmented basis sets these problems disappear allowing for the standard $X^{-3}$ extrapolation. One may also note that the relativistic corrections to the dispersion coefficients are relatively much larger than in the case of static polarizability. As the final values we take

$$
\begin{array}{ll}
\alpha_{2}^{(2)}\left(P_{4}\right)=0.0619(16), & \alpha_{2}^{(2)}\left(D_{1}\right)=-0.0479(1), \\
\alpha_{4}^{(2)}\left(P_{4}\right)=0.1799(23), & \alpha_{4}^{(2)}\left(D_{1}\right)=-0.1332(21) .
\end{array}
$$

The above results were obtained by $X^{-3}$ extrapolation from the $X=4,5$ pair of basis sets and the error was estimated as a difference between the extrapolated result and the value calculated with the $X=5$ basis set. The total relativistic correction to the dispersion coefficients $\alpha_{2}$ and $\alpha_{4}$ equals $\alpha_{2}^{(2)}=0.0141(10)$ and $\alpha_{4}^{(2)}=0.0467(1)$, respectively. The estimated errors are smaller than of the individual D1 and P4 corrections given above because in the present case we directly extrapolated the sum of the P4 and D1 corrections exploiting a systematic error cancellation.

The two-electron relativistic corrections to the static polarizability are shown in Table VIII They were obtained with help of aug-cc-pVXZ Gaussian basis sets, $X=2-5$ [35, 57] which were additionally uncontracted in these calculations. In the case of the Darwin correction, we apply the $X^{-1}$ extrapolation formula 
TABLE VIII. Two-electron relativistic corrections to the static polarizability of the neon atom calculated at the CCSD(T) level of theory with the finite-field approach.

\begin{tabular}{lccccccccc}
\hline \hline basis set & \multicolumn{3}{c}{ s-aug } & \multicolumn{3}{c}{ d-aug } & & \multicolumn{2}{c}{ t-aug } \\
\hline & $\alpha_{0}^{(2)}\left(D_{2}\right)$ & $\alpha_{0}^{(2)}(B)$ & sum & $\alpha_{0}^{(2)}\left(D_{2}\right)$ & $\alpha_{0}^{(2)}(B)$ & sum & $\alpha_{0}^{(2)}\left(D_{2}\right)$ & $\alpha_{0}^{(2)}(B)$ & sum \\
\hline cc-pVDZ & 0.000001 & 0.000794 & 0.000794 & 0.000059 & 0.001262 & 0.001321 & 0.000060 & 0.001277 & 0.001337 \\
cc-pVTZ & 0.000037 & 0.001031 & 0.001068 & 0.000063 & 0.001286 & 0.001349 & 0.000063 & 0.001290 & 0.001353 \\
cc-pVQZ & 0.000046 & 0.001175 & 0.001221 & 0.000055 & 0.001281 & 0.001336 & 0.000055 & 0.001281 & 0.001336 \\
cc-pV5Z & 0.000045 & 0.001239 & 0.001284 & 0.000048 & 0.001279 & 0.001327 & 0.000048 & 0.001279 & 0.001327 \\
\hline \hline
\end{tabular}

which gives

$$
\alpha_{0}^{(2)}\left(D_{2}\right)=0.00002(1)
$$

This formula is based on analytic results for the helium atom [68] and has been used successfully in numerous studies for larger systems 69 74]. Clearly, this correction is very small and extrapolates to nearly zero. In the case of the Breit correction, we found that there is no need to extrapolate the results. The values of $\alpha_{0}^{(2)}(B)$ are remarkably stable with respect to the size of the basis set, as shown in Table VIII, and this gives us the following estimate

$$
\alpha_{0}^{(2)}(B)=0.00128(1)
$$

Therefore, the total two-electron relativistic contribution to the polarizability of neon atom amounts to $0.00130(2)$. It seems that there are no literature results for neon that we could confront this value with.

The data provided above reveal a substantial mutual cancellation of individual contribution to the total relativistic corrections $\alpha_{n}^{(2)}$. For example, the total, i.e., including both one- and two-electron contributions, relativistic correction to $\alpha_{0}$ is about 0.0055 while the largest of these contributions (the mass-velocity term) is 0.02149. In the case of the dispersion coefficients, a similar phenomenon occurs but is somewhat less pronounced. This can be contrasted with the analogous data for the helium atom (see Table VIII in Ref. [20]) where the cancellation is very strong, especially for higher values of $n$.

Next we consider the leading-order quantum electrodynamics (QED) corrections; they shall be denoted as $\alpha_{n}^{(3)}$, $n=0,2,4$, further in the text. The correction applied in this work reads 75 77]

$$
\alpha_{n}^{(3)}=\frac{8}{3 \pi c}\left(\frac{19}{30}+2 \ln c-\ln k_{0}\right) \alpha_{n}^{(2)}\left(D_{1}\right),
$$

where $\ln k_{0}$ is the so-called Bethe logarithm [60, 78]. In comparison with the full QED treatment that has recently been reported for the helium atom [21], formula (9) contains two simplifications. First, the two-electron terms are omitted in $\hat{H}_{\mathrm{QED}}$; this is justified because they are expected to be at least several times smaller than the dominant correction (9). Second, the electric field dependence of the Bethe logarithm is neglected. Calculation of the second electric field derivative of $\ln k_{0}$ is very challenging and has been accomplished thus far only for the helium atom [17, 21]. Moreover, this derivative (in atomic units) was found to be two orders of magnitude smaller than the zero-field Bethe logarithm and thus constitutes a negligible correction. The reason for this unexpected behavior can be understood by noting that the value of the Bethe logarithm is sensitive primarily to the region of the wavefunction close to the nucleus. This regime is dominated by the electric field generated by the nucleus which is much stronger than the (perturbative) external fields.

The Bethe logarithm for the neon atom without external electric field was calculated at the Hartree-Fock level of theory within a basis set of Slater-type orbitals that includes $1 p$ functions. Details of these calculations will be described in a separate publication. The final result is $\ln k_{0}=7.595$ and we estimate that this value is accurate to within $1-2 \%$. This assumption is based on comparisons of analogous results for lighter atoms for which more accurate reference data are available. By using the value of the one-electron Darwin corrections given earlier, we obtain the following values of the QED correction to the static polarizability

$$
\alpha_{0}^{(3)}=-0.00031(6),
$$

and to the dispersion coefficients

$$
\alpha_{2}^{(3)}=-0.00085(17), \quad \alpha_{4}^{(3)}=-0.0024(5),
$$

where we have assumed an uncertainty of $20 \%$ that accounts for all approximations discussed above. It turns out that in the case of the static polarizability, this correction is not negligible within the present accuracy standards.

To estimate the contribution of the higher-order relativistic and QED effects, the so-called one-loop correction [79] was calculated. This is straightforward because for atomic systems this correction to the polarizability can be expressed as

$$
\alpha_{n}^{(4)}=\frac{2 Z}{c^{2}}\left[\frac{427}{96}-2 \ln 2\right] \alpha_{n}^{(2)}\left(D_{1}\right),
$$

and thus the computation of this correction amounts to scaling the values of one-electron Darwin corrections 
given above. If one conservatively assumes that the error of neglecting all other higher-order QED diagrams is less than $50 \%$, estimations $\alpha_{0}^{(4)}=-0.00006(3)$ is obtained for the static polarizability, and $\alpha_{2}^{(4)}=-0.00016(8)$, $\alpha_{4}^{(4)}=-0.00043(21)$ for the dispersion coefficients. This clearly indicates that higher-order relativistic and QED effects are negligible from the point of view of the present work and that the perturbative series of QED corrections is rapidly convergent.

\section{Finite nuclear mass and size corrections to the polarizability}

We also considered the finite nuclear size (FNS) and finite nuclear mass (FNM) corrections, denoted $\alpha_{n}^{\mathrm{FNS}}$ and $\alpha_{n}^{\mathrm{FNM}}$ further in the text. For neon atom, the former correction can be obtained from the formula

$$
\alpha_{n}^{\mathrm{FNS}}=\frac{4}{3} \frac{\left\langle r_{c}^{2}\right\rangle}{\hbar^{2}} \alpha_{n}^{(2)}\left(D_{1}\right),
$$

see e.g., Ref. [80], where $\left\langle r_{c}^{2}\right\rangle$ is the averaged square of the nuclear charge radius and $\lambda \approx 386.2 \mathrm{fm}$ is the reduced Compton wavelength of the electron. For the ${ }^{20} \mathrm{Ne}$ nuclide, the value $\left\langle r_{c}^{2}\right\rangle \approx 8.952 \mathrm{fm}^{2}$ was taken from the literature [81]. This gives $\alpha_{0}^{\mathrm{FNS}} \approx 1.4 \cdot 10^{-6}$ revealing that the FNS correction changes the static polarizability of the neon atom by only about 1 ppm and thus it is entirely negligible compared to other sources of error. The same conclusion holds for the dispersion coefficients.

Moving to the FNM effects, let us consider first the static polarizability. The leading-order FNM correction is composed of two terms 15 .

$$
\alpha_{0}^{\mathrm{FNM}}=3 \frac{m_{\mathrm{e}}}{m_{N}} \alpha_{0}+\frac{m_{\mathrm{e}}}{m_{N}} \partial_{\mathcal{E}}^{2}\left\langle\sum_{i \neq j} \nabla_{i} \nabla_{j}\right\rangle,
$$

where $m_{\mathrm{e}}$ and $m_{N}$ are the electron and the nuclear masses, respectively, and $\partial_{\mathcal{E}}^{2}\left\langle\sum_{i \neq j} \nabla_{i} \nabla_{j}\right\rangle$ denotes the second electric field derivative of the expectation value $\left\langle\sum_{i \neq j} \nabla_{i} \nabla_{j}\right\rangle$ evaluated at zero field (mass-polarization term). The first term of the above expression (resulting from the reduced-mass scaling) amounts to $0.00022(1)$ for ${ }^{20} \mathrm{Ne}$. The mass-polarization term is usually about an order of magnitude smaller than the mass-scaling term [15] and thus can be neglected here. The reduced-mass scaling applied the dispersion coefficients leads to

$$
\alpha_{n}^{\mathrm{FNM}} \approx(n+3) \frac{m_{\mathrm{e}}}{m_{N}} \alpha_{n}
$$

Neglecting the mass-polarization contribution, we find that $\alpha_{2}^{\mathrm{FNM}}=0.00039(1)$ and $\alpha_{4}^{\mathrm{FNM}}=0.00094(1)$.

\section{Final error budget of the polarizability calculations}

The final theoretical results for the static polarizability and dispersion coefficients of the neon atom are sum-

\begin{tabular}{|c|c|c|c|}
\hline & $\alpha_{0}$ & $\alpha_{2}$ & $\alpha_{4}$ \\
\hline \multicolumn{4}{|c|}{ clamped-nuclei non-relativistic contributions } \\
\hline unrelaxed CC3 & $2.66172(18)$ & $2.846(5)$ & $4.912(6)$ \\
\hline triples corr. & $0.00034(5)$ & - & - \\
\hline quadruples corr. & $-0.00614(17)$ & - & - \\
\hline FCI correction & $-0.00047(24)$ & $-0.009(4)$ & $-0.026(13)$ \\
\hline \multicolumn{4}{|c|}{ other contributions } \\
\hline one-el. relativistic & $0.00420(5)$ & $0.014(1)$ & $0.047(1)$ \\
\hline two-el. relativistic & $0.00130(2)$ & - & - \\
\hline leading-order QED & $-0.00031(6)$ & $-0.001(1)$ & $-0.002(1)$ \\
\hline high-order QED & $-0.00006(3)$ & $0.000(1)$ & $0.000(1)$ \\
\hline finite nuclear mass & $0.00022(1)$ & $0.000(1)$ & $0.001(1)$ \\
\hline finite nuclear size & $0.00000(1)$ & - & - \\
\hline total & $2.66080(36)$ & $2.850(7)$ & $4.932(14)$ \\
\hline
\end{tabular}

TABLE IX. Final error budget of the calculations of the static polarizability and dispersion coefficients for the neon atom.

marized in Table IX. We show the magnitude of various contributions to $\alpha_{n}$ along with their error estimation, according to the discussion from the previous sections. The final error is obtained by summing the errors in the individual components quadratically. Clearly, the dominant source of error is the insufficiently accurate description of higher-order excitations, particularly of the FCI correction. In fact, with our resources it would still probably be possible (albeit at a huge cost) to perform CC3 calculations with the cardinal number $X$ of the basis set size increased by one. Coupled with the CBS extrapolation, which was shown to perform quite well at the CC3 level of theory, this may lead to the error reduction by a factor of about two in the CC3 component of the polarizability. However, this would not decrease the overall uncertainty of our result since already at the present level it is dominated by the error of the FCI correction.

Rather surprisingly, the relativistic corrections to $\alpha_{n}$ can be calculated with a sufficient accuracy. While they are extremely complicated from the point of view of analytical evaluation, it appears that the mixed analytic and finite-field approach adopted by us is adequate. It is also worth mentioning that the approximations adopted by us in the computations of the QED corrections do not contribute significantly to the overall error. The errors resulting from omission of the two-electron QED contributions and from missing electric-field derivative of the Bethe logarithm would become important only when other sources of error were reduced by a factor of at least five.

The data provided in Table IX also reveal a substantial cancellation between various contributions to the static polarizability. Indeed, the one- and two-electron relativistic corrections combined amount to about 0.0053 and are of opposite sign to the total nonrelativistic post-CC3 correction which is equal to -0.0063 . This means that the difference between the pure CC3 result and the ex- 
TABLE X. Expectation values $\left\langle\Sigma_{i} r_{i}^{2}\right\rangle$ calculated for the neon atom. The valence and core correlation contributions were obtained at the CC3/(aug-)cc-pVXZ and CC3/(aug-)cc-pCVXZ levels of theory, respectively.

\begin{tabular}{cccccc}
\hline \hline & $\mathrm{DZ}$ & $\mathrm{TZ}$ & $\mathrm{QZ}$ & $5 \mathrm{Z}$ & $6 \mathrm{Z}$ \\
\hline \multicolumn{7}{c}{ valence contribution } \\
\hline no-aug & 9.1422 & 9.3894 & 9.4929 & 9.5544 & 9.5661 \\
aug & 9.7022 & 9.6336 & 9.5994 & 9.5802 & 9.5740 \\
\hline \multicolumn{7}{c}{ core correlation } & contribution \\
\hline no-aug & -0.0017 & -0.0062 & -0.0079 & -0.0088 & - \\
aug & -0.0026 & -0.0071 & -0.0085 & -0.0089 & - \\
\hline \hline
\end{tabular}

perimental vale is much smaller than one could expect a priori. The same effect is present also in calculations of the dispersion coefficients, albeit to a smaller extent. This may also explain an unexpectedly good agreement of some literature values with the experimental results despite the fact that all post-CC3/post-CCSD(T) and relativistic corrections were neglected.

\section{E. Magnetic susceptibility}

As mentioned in Sec. [. the determination of temperature or pressure via measurements of the refractive index $n$ requires also the knowledge of the magnetic susceptibility, $\chi$. For the neon atom this quantity is about $3 \cdot 10^{-5}$ times smaller in magnitude than the static polarizability [82]. Therefore, if the required relative accuracy in calculation of the refractive index is of the order of $10^{-6}$, it is sufficient to know $\chi$ with an uncertainty of only 1 percent, as evident from the Clausius-Mossotti relations for $\epsilon_{r}$ and $\mu_{r}$. This allows us to adopt significant simplifications in evaluation of the magnetic susceptibility, namely (i) the frequency dependence of $\chi$ can be entirely neglected and (ii) the static value of $\chi_{0}$ can be calculated from the "non-relativistic" formula [60],

$$
\chi_{0}=-\frac{e^{2}}{6 m_{\mathrm{e}} c^{2}}\left\langle\sum_{i} r_{i}^{2}\right\rangle
$$

where $\left\langle r_{i}^{2}\right\rangle$ is the mean square distance of an electron from the nucleus.

The quantity $\left\langle\sum_{i} r_{i}^{2}\right\rangle$ was computed directly as an expectation value with the CC3 wavefunction using the formalism proposed by Tucholska et al. [83] In this method one introduces an auxiliary excitation operator $S$ [84], defined by a closed-form linear equation involving the standard cluster operator $T$ and its Hermitian conjugate. With help of the $S$ operator one can rewrite the $\mathrm{CC}$ expectation value of an arbitrary operator as a finite commutator expansion. Since the exact $S$ operator corresponding to the $\mathrm{CC} 3$ wavefunction involves excitation levels higher than triple, it is necessary to truncate it for practical reasons. In this work we employ the truncation at the third-order of perturbation theory as suggested in Ref. 83] which provides an optimal balance between the accuracy and computational costs.

The valence results, i.e., with the frozen $1 s^{2}$ core, were obtained at the CC3/(aug-)cc-pVXZ level of theory. For the evaluation of the core correlation contribution we used the extended (aug-)cc-pCVXZ basis sets that include additional tight functions (with large exponents) for a better description of the core region. The results are shown in Table $\mathrm{X}$. Both the valence and core contributions were extrapolated to the complete basis set limit from the largest two augmented basis sets using the $X^{-3}$ formula. In each case, we determine the error as a difference between the extrapolated and the largest basis set results. This gives us 9.565(9) and -0.009(1) for the valence and core correlation contributions, respectively. Concerning the augmentation level, we found the single augmentation to be entirely sufficient for the calculation of $\left\langle\sum_{i} r_{i}^{2}\right\rangle$. We checked that already at the quadruplezeta level, the inclusion of the second set of diffuse functions changes the results shown in Table $\mathrm{X}$ only at the last significant digit. Finally, we add the FCI correction that we managed to compute only with aug-cc-pVDZ and aug-cc-pVTZ basis sets, obtaining 0.00148 and 0.00170 , respectively. By extrapolating these two values using the $X^{-3}$ formula, we obtain our final estimation of the FCI correction, equal to 0.0018(1). By combining the valence, core correlation, and FCI contributions and adding the errors quadratically we find

$$
\left\langle\sum_{i} r_{i}^{2}\right\rangle=9.558(10)
$$

which is our final value of the mean square distance of the electrons from the nucleus. The "nonrelativistic" diamagnetic susceptibility of the neon atom is thus

$$
\chi_{0}=-8.483(8) \cdot 10^{-5} \text { a.u. }
$$

or $\chi_{0}=-7.570(8) \cdot 10^{-6} \mathrm{~cm}^{3} / \mathrm{mol}$ in units used conventionally in experimental work.

Below we consider corrections to this result that account for effects not included in the "nonrelativistic" approximation of Eq. (14). The finite-nuclear-mass correction, $\chi_{0}^{\mathrm{FNM}}$, to the magnetic susceptibility was considered by Bruch and Weinhold [85] for the helium atom, see also Ref. [86] for an erratum to this work. These authors derived the complete formula, including the masspolarization term, for the leading contribution to $\chi_{0}^{\mathrm{FNM}}$ of the order of $m_{\mathrm{e}} / m_{\mathrm{N}}$. They found that the masspolarization correction is by about 5 orders of magnitude smaller than $\chi_{0}$. For the neon atom, this ratio will be even smaller because of much larger mass of the ${ }^{20} \mathrm{Ne}$ nucleus. Therefore, in our work we neglected the masspolarization contribution to $\chi_{0}$. The derivation of Bruch and Weinhold was recently generalized to many-electron atoms by Pachucki and Yerokhin [87]. When the mass- 
polarization term is neglected, their formula reads

$$
\chi_{0}^{\mathrm{FNM}}=-\frac{e^{2}}{2 m_{N} c^{2}}\left\langle\sum_{i} r_{i}^{2}\right\rangle-\frac{e^{2}}{6 m_{N} c^{2}}\left\langle\sum_{i \neq j} \mathbf{r}_{i} \cdot \mathbf{r}_{j}\right\rangle .
$$

The first term is simply a scaling of the value of Eq. (16) by a factor of $3 m_{\mathrm{e}} / m_{N}$. This gives a correction equal to $-6.98 \cdot 10^{-9}$ a.u. which is not entirely negligible in the present context.

Bruch and Weinhold [85, 86] found that the second term in Eq. (17), i.e., involving the operator $\sum_{i \neq j} \mathbf{r}_{i} \cdot \mathbf{r}_{j}$, is very small, even smaller than the mass-polarization term. However, unlike for helium, in the case of neon the expectation value $\left\langle\sum_{i \neq j} \mathbf{r}_{i} \cdot \mathbf{r}_{j}\right\rangle$ does not vanish at the uncorrelated, i.e., at the Hartree-Fock level, due to the presence of the $p$ orbitals in the ground-state reference determinant. Therefore, the estimation based on the helium results may not be reliable and we decided to calculate this term in an approximate way to remove this potential source of uncertainty. For this purpose we used the CC3 wavefunction and calculated the two-electron expectation value in the leading order of the perturbation theory 84]. Using the aug-cc-pCV5Z basis set and with all electrons correlated, we found that

$$
\left\langle\sum_{i \neq j} \mathbf{r}_{i} \cdot \mathbf{r}_{j}\right\rangle=-3.614
$$

This expectation value turns out to be quite large, only about two and a half times smaller than the one-electron counterpart, $\left\langle\sum_{i} r_{i}^{2}\right\rangle$. Still, the second term in Eq. (17) amounts to only $8.8 \cdot 10^{-10}$ a.u. and can be neglected even in computing the total uncertainty of $\chi$. The inclusion of the finite nuclear mass corrections in calculation of the magnetic susceptibility of the neon atom will be necessary only if one aims at achieving the accuracy level of $10^{-4}$ or better.

Let us finally consider the relativistic correction to $\chi_{0}$. The leading term in this correction is of the order of $1 / c^{4}$ and will be denoted by $\chi_{0}^{(4)}$. Complete computation of $\chi_{0}^{(4)}$ for a noble gas atom represents a considerable challenge and has not been performed thus far even for helium. In their helium study, Bruch and Weinhold [85] neglected the magnetic-field dependent terms in the Breit-Pauli Hamiltonian [86, 88] and found that the computed approximation to $\chi_{0}^{(4)}$ is by a factor of $8 \cdot 10^{-5}$ smaller than nonrelativistic value of $\chi_{0}$ and, therefore, negligible for this system. Since the ratio $\chi_{0}^{(4)} / \chi_{0}$ scales quadratically with an effective nuclear charge $Z_{\text {eff }}$, the significance of $\chi_{0}^{(4)}$ must certainly be much larger for neon then for helium. Any choice of $Z_{\text {eff }}$ would be to a large extent arbitrary, so we decided to estimate $\chi_{0}^{(4)}$ assuming that the significance of the relativistic correction to $\chi_{0}$ is percentagewise the same as in the case of the relativistic correction to $\alpha_{0}$. From Table IX we see that $\alpha_{0}^{(2)}=0.00550(6)$ represents $0.2 \%$ of $\alpha_{0}$. Assuming
TABLE XI. Comparison with other theoretical and experimental literature values of $\alpha_{n}$. Wherever no error estimation is present it means that they were not given by the authors.

\begin{tabular}{|c|c|c|c|}
\hline & $\alpha_{0}$ & $\alpha_{2}$ & $\alpha_{4}$ \\
\hline \multicolumn{4}{|c|}{ experimental } \\
\hline Chan et al. [89] & - & 2.938 & 5.137 \\
\hline Kumar and Meath [29] & 2.669 & 2.875 & 4.994 \\
\hline \multirow[t]{2}{*}{ Orcutt and Cole [90] } & 2.658 & - & - \\
\hline & $2.663^{\mathrm{a}}$ & - & - \\
\hline Gaiser and Fallmuth [91] & $2.66110(3)$ & - & - \\
\hline Gaiser and Fallmuth [7] & $2.661057(7)$ & - & - \\
\hline \multicolumn{4}{|c|}{ theoretical } \\
\hline Pawłowski et al. [26] & 2.665 & 2.859 & 4.946 \\
\hline Klopper et al. [22] & 2.66312 & - & - \\
\hline Larsen et al. [23] & 2.673 & - & - \\
\hline \multicolumn{4}{|c|}{ this work } \\
\hline this work & $2.66080(36)$ & $2.850(7)$ & $4.932(14)$ \\
\hline
\end{tabular}
All values are given in the atomic units.

${ }^{\mathrm{a}}$ after applying the correction for the compressibility

the same proportion for the magnetic susceptibility, we find that that the magnitude of $\chi_{0}^{(4)}$ can be estimated as $0.017 \cdot 10^{-5}$ and we add this value to the final error budget of $\chi_{0}$. This is sufficient for the present purpose but it is clear that the complete calculation of the relativistic corrections to the magnetic susceptibility of helium and other noble gases represents interesting topic for a future study.

\section{DISCUSSION AND CONCLUSIONS}

In Table XI, we compare the results of polarizability calculations with other theoretical work and experimental values taken from the literature. While our intention was to include the most recent and representative results available currently, we by no means claim this list to be exhaustive.

Our final theoretical value of the static polarizability $\alpha_{0}=2.66080(36)$ has an estimated uncertainty of about one part per $10^{4}$. The most recent experimental value for this quantity, $\alpha_{0}=2.661057(7)$, obtained by Gaiser and Fellmuth [7], lies within our error bars, but is about 50 times more accurate. Therefore, unlike for helium, the present-day theoretical methods for ten-electron atoms are not competitive in terms of accuracy with the results obtained from the dielectric-constant thermometry experiments. Nonetheless, it is clear from Table XI that our results are the most reliable theoretical data available for $\alpha_{0}$, especially taking into account the systematic inclusion of various small physical effects and more rigorous error estimations. The best previous theoretical estimate for $\alpha_{0}$ presented in Ref. 7] is obtained by combining the relativistic results of Klopper et al. [22] with 
the FCI correction calculated by Larsen et al. [23] resulting in $\alpha_{0}=2.6617(20)$. The estimated uncertainty of this value is about six times larger than the uncertainty of our result. One may note that the largest contribution to our error budget comes from the approximate (small basis set) FCI treatment of $n$-electron excitations, $n>4$. A significant reduction of this error will be difficult because of the exponential scaling of the time and memory resources needed to perform FCI calculations.

The reliability of the experimental results is less impressive in the case of the dispersion coefficients. The older data of Chan et al. [89] are significantly less accurate than our values. The most recent data come from the work of Kumar and Thakkar 29] who employed a semiempirical dipole oscillator strength distribution (DOSD) technique to extract $\alpha_{n}$ from the experimental photoabsorption cross sections with addition of some theoretical constraints. These authors estimated that their results for $\alpha_{2}$ and $\alpha_{4}$ are accurate to about $\pm 1 \%$ and, therefore, agree to within the combined uncertainty estimates with the values calculated here. Similarly, our results also agree well with the theoretical data of Pawłowski et al. [26] who also estimate a similar accuracy level. Note that the dispersion coefficients calculated in the present work $\left[\alpha_{2}=2.850(7)\right.$ and $\left.\alpha_{4}=4.932(14)\right]$ are estimated to be accurate to about one-two parts per thousand. Therefore, they are by almost an order of magnitude more accurate than the best previous estimates, including both theoretical and experimental literature data.

The value of the static magnetic susceptibility $\chi_{0}$ obtained by us is $-8.484(19) \cdot 10^{-5}$ a.u., or $-7.571(17)$. $10^{-6} \mathrm{~cm}^{3} / \mathrm{mol}$, where the reported uncertainties include now the entire value of the estimated relativistic correction. Our result, estimated to be accurate to about 2 parts per thousand, agrees quite well with the older experimental value reported by Havens [92] equal to $-7.651 \cdot 10^{-6} \mathrm{~cm}^{3} / \mathrm{mol}$, but is about $9 \%$ larger in absolute value than the more recent recommended experimental result of $-6.96 \cdot 10^{-6} \mathrm{~cm}^{3} / \mathrm{mol}$ [82, 93] One may note that also for helium there is about $7 \%$ discrepancy between the best theoretical result [85] and the recommended experimental value [82]. The reasons behind these differences between theory and experiment are not clear. In the case of neon, one can consider the following hypothetical sources of the observed discrepancy:

(a) significant underestimation of relativistic correction to $\chi_{0}$,

(b) neglect of the paramagnetic contribution to $\chi_{0}$,

(c) neglect of the temperature dependence of $\chi_{0}$,

(c) neglect of the density dependence of $\chi_{0}$.

The source $(a)$ is unlikely since the relativistic correction included in our uncertainty estimate represents only about $0.2 \%$ of $\chi_{0}$. This value is approximately 25 times larger than the estimate obtained for helium, suggesting that the ratio $Z_{\text {eff }}(\mathrm{Ne}) / Z_{\text {eff }}(\mathrm{He})$ is about 5 . Even if this estimation is multiplied by a factor of 4 , which corresponds to assuming the maximal possible value of the ratio $Z_{\text {eff }}(\mathrm{Ne}) / Z_{\text {eff }}(\mathrm{He})=10$, the experimental value remains $10 \sigma$ away from our theoretical result.

Regarding point $(b)$, we found that the leading nonrelativistic paramagnetic contribution to $\chi_{0}$ is of the order of $\left(m_{\mathrm{e}} / m_{\mathrm{N}}\right)^{2}$ and is therefore entirely negligible. This contribution was considered in the Appendix of Ref. 85. and incorrectly claimed to vanish for helium. The argument given in this Appendix is based on the assumption that quantum states of the helium atom cannot have ${ }^{1} P^{\mathrm{e}}$ symmetry, which is incorrect in the continuous spectrum. There is also relativistic paramagnetic contribution to $\chi_{0}$ resulting from the relativistic ${ }^{3} P_{0}$ component of the ground-state helium wave function. This contribution is of the order of $1 / c^{6}$ and is completely negligible. One may note here that the frequency dependence of $\chi$ may result only from paramagnetic terms. The arguments given above show that this frequency dependence is extremely weak for rare gases and entirely negligible in practice.

Regarding point $(c)$, one can observe that for an atom moving in the magnetic field, the center of mass cannot be separated and the magnetic susceptibility depends on the momentum of the atom. This effect was considered by Bruch and Weinhold in Ref. [85] and found to be four orders of magnitude smaller than the finite nuclear mass correction $\chi_{0}^{\mathrm{FNM}}$ (for ${ }^{3} \mathrm{He}$ at $T \approx 10$ kelvin). For neon this correction will be even smaller because of its greater mass and can be assumed negligible at normal temperatures.

The point $(d)$, that is the density dependence, was considered by Bruch and Weinhold 94] for liquid helium. These authors used a simple Hartree-Fock plus dispersion model for the interaction-induced magnetic susceptibility of helium and found that the effect of the interatomic interactions is small and the measurement in the liquid can be used to accurately determine the isolated atom magnetic susceptibility. This conclusion must obviously apply also to the gas phase. Since the measurement for neon was made in the gas phase, the density dependence of the magnetic susceptibility cannot explain the observed difference between our theoretical value and the experimental one. The source of this difference remains unclear to us and represents, as for helium, a troubling puzzle to be hopefully resolved in the future by new theoretical ideas or new experiments.

To sum up, we have reported state-of-the-art theoretical determination of the static and dynamic polarizability of the neon atom. Numerous corrections beyond the nonrelativistic clamped-nuclei picture, such as those due to the quantum electrodynamics effects, have been included for the first time for this system and the sources of error have been carefully discussed. Additionally, we determined the magnetic susceptibility of the neon atom with an accuracy of a few parts per thousand. Since the magnetic contribution to the refractive index of the gaseous neon is about five orders of magnitude smaller than the very accurately known electric contribution, our result is 
entirely sufficient to calculate the refractive index with an accuracy of one part per million.

\section{ACKNOWLEDGMENTS}

We thank Krzysztof Pachucki for discussions and Christof Gaiser, Roberto Gavioso, and Krzysztof Szalewicz for comments on the manuscript. This project
(QuantumPascal project 18SIB04) has received funding from the EMPIR programme cofinanced by the Participating States and from the European Union's Horizon 2020 research and innovation program. The authors also acknowledge support from the National Science Center, Poland, within the Project No. 2017/27/B/ST4/02739. ML was supported by the Foundation for Polish Science (FNP) and by the Polish National Agency of Academic Exchange through the Bekker programme No. PPN/BEK/2019/1/00315/U/00001.
[1] B. Song and Q.-Y. Luo, Metrologia 57, 025007 (2020).

[2] C. Gaiser, B. Fellmuth, and T. Zandt, Int. J. Thermophys. 35, 395 (2014).

[3] C. Gaiser, T. Zandt, and B. Fellmuth, Metrologia 52, S217 (2015).

[4] C. Guenz, C. Gaiser, and M. Richter, Meas. Sci. Technol. 28, 027002 (2017).

[5] C. Gaiser and B. Fellmuth, J. Chem. Phys. 150, 134303 (2019).

[6] C. Gaiser, B. Fellmuth, and W. Sabuga, Nat. Phys. 16, 177 (2020).

[7] C. Gaiser and B. Fellmuth, Phys. Rev. Lett. 120, 123203 (2018).

[8] J. W. Schmidt, R. M. Gavioso, E. F. May, and M. R. Moldover, Phys. Rev. Lett. 98, 254504 (2007).

[9] K. Jousten, J. Hendricks, D. Barker, K. Douglas, S. Eckel, P. Egan, J. Fedchak, J. Flügge, C. Gaiser, D. Olson, J. Ricker, T. Rubin, W. Sabuga, J. Scherschligt, R. Schödel, U. Sterr, J. Stone, and G. Strouse, Metrologia 54, S146 (2017).

[10] J. Hendricks, Nat. Phys. 14, 100 (2018).

[11] J. Fischer, Ann. Phys. 531, 1800304 (2019).

[12] G. Machin, IEEE Instru. Meas. Mag. 22, 17 (2019).

[13] W. R. Johnson and K. T. Cheng, Phys. Rev. A 53, 1375 (1996).

[14] A. K. Bhatia and R. J. Drachman, Phys. Rev. A 58, 4470 (1998).

[15] K. Pachucki and J. Sapirstein, Phys. Rev. A 63, 012504 (2000).

[16] W. Cencek, K. Szalewicz, and B. Jeziorski, Phys. Rev. Lett. 86, 5675 (2001).

[17] G. Łach, B. Jeziorski, and K. Szalewicz, Phys. Rev. Lett. 92, 233001 (2004).

[18] M. Puchalski, U. D. Jentschura, and P. J. Mohr, Phys. Rev. A 83, 042508 (2011).

[19] K. Piszczatowski, M. Puchalski, J. Komasa, B. Jeziorski, and K. Szalewicz, Phys. Rev. Lett. 114, 173004 (2015).

[20] M. Puchalski, K. Piszczatowski, J. Komasa, B. Jeziorski, and K. Szalewicz, Phys. Rev. A 93, 032515 (2016).

[21] M. Puchalski, K. Szalewicz, M. Lesiuk, and B. Jeziorski, Phys. Rev. A 101, 022505 (2020).

[22] W. Klopper, S. Coriani, T. Helgaker, and P. Jørgensen, J. Phys. B: At. Mol. Opt. Phys. 37, 3753 (2004).

[23] H. Larsen, J. Olsen, C. Hättig, P. Jørgensen, O. Christiansen, and J. Gauss, J. Chem. Phys. 111, 1917 (1999).

[24] R. Franke, H. Müller, and J. Noga, J. Chem. Phys. 114, 7746 (2001).

[25] K. Hald, F. Pawłowski, P. Jørgensen, and C. Hättig, J. Chem. Phys. 118, 1292 (2003).
[26] F. Pawłowski, P. Jørgensen, and C. Hattig, in Adv. Quantum Chem., Vol. 48 (Academic Press, 2005) pp. $9-$ 21.

[27] V. E. Chernov, D. L. Dorofeev, I. Y. Kretinin, and B. A. Zon, Phys. Rev. A 71, 022505 (2005).

[28] J. R. Hammond, W. A. de Jong, and K. Kowalski, J. Chem. Phys. 128, 224102 (2008).

[29] A. Kumar and A. J. Thakkar, J. Chem. Phys. 132, 074301 (2010).

[30] E. Tiesinga, P. J. Mohr, D. B. Newell, and B. N. Taylor, The 2018 CODATA Recommended Values of the Fundamental Physi (2018, accessed September 3, 2020), available online at http://physics.nist.gov/constants.

[31] M. Wang, G. Audi, F. G. Kondev, W. Huang, S. Naimi, and X. Xu, Chin. Phys. C 41, 030003 (2017).

[32] E. B. Saloman and C. J. Sansonetti, J. Phys. Chem. Ref. Data 33, 1113 (2004).

[33] J. C. Slater, Phys. Rev. 36, 57 (1930).

[34] J. C. Slater, Phys. Rev. 42, 33 (1932).

[35] T. H. Dunning, J. Chem. Phys. 90, 1007 (1989).

[36] M. Lesiuk, M. Przybytek, M. Musiał, B. Jeziorski, and R. Moszynski, Phys. Rev. A 91, 012510 (2015).

[37] M. Lesiuk, A. M. Tucholska, and R. Moszynski, Phys. Rev. A 95, 052504 (2017).

[38] M. Lesiuk and R. Moszynski, Phys. Rev. E 90, 063318 (2014).

[39] M. Lesiuk and R. Moszynski, Phys. Rev. E 90, 063319 (2014).

[40] M. Lesiuk, J. Math. Chem. 54, 572 (2016).

[41] R. J. Bartlett and M. Musial, Rev. Mod. Phys. 79, 291 (2007).

[42] Z. W. Liu and H. P. Kelly, Theor. Chem. Acc. 80, 307 (1991).

[43] B. K. Sahoo and B. P. Das, Phys. Rev. A 77, 062516 (2008).

[44] G. D. Purvis and R. J. Bartlett, J. Chem. Phys. 76, 1910 (1982).

[45] G. E. Scuseria, A. C. Scheiner, T. J. Lee, J. E. Rice, and H. F. Schaefer, J. Chem. Phys. 86, 2881 (1987).

[46] R. N. Hill, J. Chem. Phys. 83, 1173 (1985).

[47] T. Helgaker, W. Klopper, H. Koch, and J. Noga, J. Chem. Phys. 106, 9639 (1997).

[48] A. Halkier, T. Helgaker, P. Jørgensen, W. Klopper, H. Koch, J. Olsen, and A. K. Wilson, Chem. Phys. Lett. 286, 243 (1998).

[49] K. Aidas, C. Angeli, K. L. Bak, V. Bakken, R. Bast, L. Boman, O. Christiansen, R. Cimiraglia, S. Coriani, P. Dahle, E. K. Dalskov, U. Ekström, T. Enevoldsen, J. J. Eriksen, P. Ettenhuber, B. Fernández, L. Ferrighi, 
H. Fliegl, L. Frediani, K. Hald, A. Halkier, C. Hättig, H. Heiberg, T. Helgaker, A. C. Hennum, H. Hettema, E. Hjertenæs, S. Høst, I.-M. Høyvik, M. F. Iozzi, B. Jansík, H. J. Aa. Jensen, D. Jonsson, P. Jørgensen, J. Kauczor, S. Kirpekar, T. Kjærgaard, W. Klopper, S. Knecht, R. Kobayashi, H. Koch, J. Kongsted, A. Krapp, K. Kristensen, A. Ligabue, O. B. Lutnæs, J. I. Melo, K. V. Mikkelsen, R. H. Myhre, C. Neiss, C. B. Nielsen, P. Norman, J. Olsen, J. M. H. Olsen, A. Osted, M. J. Packer, F. Pawlowski, T. B. Pedersen, P. F. Provasi, S. Reine, Z. Rinkevicius, T. A. Ruden, K. Ruud, V. V. Rybkin, P. Sałek, C. C. M. Samson, A. S. de Merás, T. Saue, S. P. A. Sauer, B. Schimmelpfennig, K. Sneskov, A. H. Steindal, K. O. Sylvester-Hvid, P. R. Taylor, A. M. Teale, E. I. Tellgren, D. P. Tew, A. J. Thorvaldsen, L. Thøgersen, O. Vahtras, M. A. Watson, D. J. D. Wilson, M. Ziolkowski, and H. Ågren, WIREs Comput. Mol. Sci. 4, 269 (2014).

[50] "DALton, a molecular electronic structure program, release 2018.alpha," (2018), see http://daltonprogram.org.

[51] J. Noga and R. J. Bartlett, J. Chem. Phys. 86, 7041 (1987).

[52] G. E. Scuseria and H. F. Schaefer, Chem. Phys. Lett. 152, 382 (1988).

[53] S. A. Kucharski and R. J. Bartlett, J. Chem. Phys. 97, 4282 (1992).

[54] D. A. Matthews and J. F. Stanton, J. Chem. Phys. 142, 064108 (2015).

[55] S. A. Kucharski and R. J. Bartlett, Chem. Phys. Lett. 158, 550 (1989).

[56] Y. J. Bomble, J. F. Stanton, M. Kállay, and J. Gauss, J. Chem. Phys. 123, 054101 (2005).

[57] R. A. Kendall, T. H. Dunning, and R. J. Harrison, J. Chem. Phys. 96, 6796 (1992).

[58] M. Kállay, P. R. Nagy, D. Mester, Z. Rolik, G. Samu, J. Csontos, J. Csóka, P. B. Szabó, L. Gyevi-Nagy, B. Hégely, I. Ladjánszki, L. Szegedy, B. Ladóczki, K. Petrov, M. Farkas, P. D. Mezei, and A. Ganyecz, J. Chem. Phys. 152, 074107 (2020).

[59] M. Przybytek, unpublished (2014), general FCI program HECTOR

[60] H. A. Bethe and E. E. Salpeter, Quantum Mechanics of One- and Two-Electron Systems (Springer: Berlin, 1975).

[61] S. Coriani, T. Helgaker, P. Jørgensen, and W. Klopper, J. Chem. Phys. 121, 6591 (2004).

[62] L. Adamowicz, W. D. Laidig, and R. J. Bartlett, Int. J. Quantum Chem. 26, 245 (1984).

[63] G. Fitzgerald, R. J. Harrison, and R. J. Bartlett, J. Chem. Phys. 85, 5143 (1986).

[64] E. A. Salter, G. W. Trucks, and R. J. Bartlett, J. Chem. Phys. 90, 1752 (1989).

[65] H. Koch, H. J. A. Jensen, P. Jørgensen, T. Helgaker, G. E. Scuseria, and H. F. Schaefer, J. Chem. Phys. 92, 4924 (1990).
[66] A. Rutkowski, J. Phys. B 19, 199 (1986).

[67] W. Kutzelnigg, Z. Phys. D 11, 15 (1989).

[68] W. Kutzelnigg, Int. J. Quantum Chem. 108, 2280 (2008).

[69] N. Middendorf, S. Höfener, W. Klopper, and T. Helgaker, Chem. Phys. 401, 146 (2012).

[70] F. A. Bischoff, E. F. Valeev, W. Klopper, and C. L. Janssen, J. Chem. Phys. 132, 214104 (2010).

[71] E. Ottschofski and W. Kutzelnigg, J. Chem. Phys. 106, 6634 (1997).

[72] M. Przybytek, W. Cencek, J. Komasa, G. Łach, B. Jeziorski, and K. Szalewicz, Phys. Rev. Lett. 104, 183003 (2010).

[73] M. Przybytek, W. Cencek, B. Jeziorski, and K. Szalewicz, Phys. Rev. Lett. 119, 123401 (2017).

[74] W. Cencek, M. Przybytek, J. Komasa, J. B. Mehl, B. Jeziorski, and K. Szalewicz, J. Chem. Phys. 136, 224303 (2012).

[75] W. Caswell and G. Lepage, Phys. Lett. B 167, 437 (1986).

[76] K. Pachucki, Ann. Phys. 226, 1 (1993).

[77] K. Pachucki, J. Phys. B 31, 5123 (1998).

[78] C. Schwartz, Phys. Rev. 123, 1700 (1961).

[79] M. I. Eides, H. Grotch, and V. A. Shelyuto, Phys. Rep. 342, 63 (2001).

[80] M. Puchalski, D. Kedziera, and K. Pachucki, Phys. Rev. A 82, 062509 (2010).

[81] H. D. Vries, C. D. Jager, and C. D. Vries, At. Data Nucl. Data Tables 36, 495 (1987).

[82] J. R. Rumble, D. R. Lide, and T. J. Bruno, $C R C$ handbook of chemistry and physics 2019-2020: a readyreference book of chemical and physical data (CRC Press, 2019).

[83] A. M. Tucholska, M. Modrzejewski, and R. Moszynski, J. Chem. Phys. 141, 124109 (2014).

[84] B. Jeziorski and R. Moszynski, Int. J. Quantum Chem. 48, 161 (1993).

[85] L. W. Bruch and F. Weinhold, J. Chem. Phys. 117, 3243 (2002)

[86] L. W. Bruch and F. Weinhold, J. Chem. Phys. 119, 638 (2003).

[87] K. Pachucki and V. A. Yerokhin, Phys. Rev. A 100, 062510 (2019).

[88] K. Pachucki, Phys. Rev. A 67, 012504 (2003).

[89] W. F. Chan, G. Cooper, X. Guo, and C. E. Brion, Phys. Rev. A 45, 1420 (1992).

[90] R. H. Orcutt and R. H. Cole, J. Chem. Phys. 46, 697 (1967).

[91] C. Gaiser and B. Fellmuth, EPL (Europhys. Lett.) 90, 63002 (2010).

[92] G. G. Havens, Phys. Rev. 43, 992 (1933).

[93] C. Barter, R. G. Meisenheimer, and D. P. Stevenson, J. Phys. Chem. 64, 1312 (1960).

[94] L. W. Bruch and F. Weinhold, J. Chem. Phys. 113, 8667 (2000). 